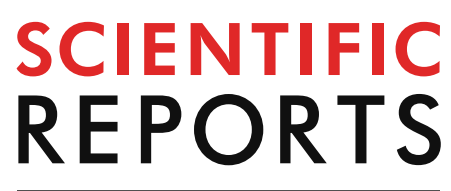

natureresearch

Check for updates

\title{
OPEN The metal binding site composition of the cation diffusion facilitator protein MamM cytoplasmic domain impacts its metal responsivity
}

\begin{abstract}
Shiran Barber-Zucker ${ }^{1,2,3}$, Anat Shahar ${ }^{2}$, Sofiya Kolusheva ${ }^{3}$ \& Raz Zarivach ${ }^{1,2,3 凶}$
The cation diffusion facilitator (CDF) is a conserved family of divalent $\mathrm{d}$-block metal cation transporters that extrude these cations selectively from the cytoplasm. CDF proteins are composed of two domains: the transmembrane domain, through which the cations are transported, and a regulatory cytoplasmic C-terminal domain (CTD). It was recently shown that the CTD of the CDF protein MamM from magnetotactic bacteria has a role in metal selectivity, as binding of different metal cations exhibits distinctive affinities and conformations. It is yet unclear whether the composition of the CTD binding sites can impact metal selectivity and if we can manipulate the CTD to response to other non-native metals in CDF proteins. Here we performed a mutational study of the model protein MamM CTD, where we exchanged the native metal binding residues with different metal-binding amino acids. Using X-ray crystallography and Trp-fluorescence spectrometry, we studied the impact of these mutations on the CTD conformation in the presence of non-native metals. Our results reveal that the incorporation of such mutations alters the domain response to metals in vitro, as mutant forms of the CTD bind metals differently in terms of the composition of the binding sites and the CTD conformation. Therefore, the results demonstrate the direct influence of the CTD binding site composition on CDF proteins structure and hence, function, and constitute a first step for rational design of MamM for transporting different metals in vivo.
\end{abstract}

Divalent $d$-block metal cations (DDMCs), such as $\mathrm{Zn}^{2+}, \mathrm{Mn}^{2+}$ and $\mathrm{Fe}^{2+}$, are crucial for numerous cell functions, hence their cellular accumulation should be effectively regulated ${ }^{1,2}$. The cation diffusion facilitator (CDF) proteins are a conserved family of DDMC transporters that extrude DDMCs from the cytoplasm through the cell membrane or into inner cellular compartments, usually by exploiting the proton motive force, and by so doing ensure the homeostasis of these cations at the cellular level ${ }^{3}$. Like other metalloproteins, each CDF protein can effectively bind and transport only specific metals ${ }^{4-7}$. In the case of CDF proteins, the metal selectivity mechanism should be highly sensitive, as these proteins control the overall concentrations of the DDMCs inside the cells and hence directly influence all metalloprotein function. CDF proteins form dimers and are typically composed of two domains ${ }^{5,8-10}$. The DDMCs are transported through the transmembrane domain via a conserved and well-defined monomeric metal binding site which also controls, to some extent, the metal selectivity ${ }^{4,11,12}$. The cytoplasmic C-terminal domain (CTD), which is frequently found in CDF proteins, forms a dimeric V-shape that enables the concerted movement of the full monomers, and serves as a regulatory domain ${ }^{5,12-14}$. In high DDMC concentrations, the DDMCs bind to specific metal binding sites in this domain, which causes a closure of the dynamic $\mathrm{V}$-shaped apo form to a rigid and tighter conformation. This specific tighter conformation, in turn, facilitates the conformational change of the TMD from a cytoplasmic-facing conformation to an outermembrane / inner-compartmental-facing conformation which allows the release of the DDMCs ${ }^{5,12-15}$. The CTD was also shown to be related with metal selectivity, which enables another level of regulation by this domain ${ }^{16}$. However, as the metal binding sites in this domain are not highly conserved, it is not clear whether the location and composition of the CTD binding sites can impact metal selectivity.

${ }^{1}$ Department of Life Sciences, Ben-Gurion University of the Negev, P.O.B. 653, 8410501 Beer Sheva, Israel. ${ }^{2}$ The National Institute for Biotechnology in the Negev, Ben-Gurion University of the Negev, 8410501 Beer Sheva, Israel. ${ }^{3}$ llse Katz Institute for Nanoscale Science and Technology, Ben-Gurion University of the Negev, 8410501 Beer Sheva, Israel. ${ }^{\boxplus}$ email: zarivach@bgu.ac.il 
MamM is a model CDF protein found in magnetotactic bacteria (MTB). MTB can sense the geomagnetic field and thereby navigate to their preferred habitats, usually oxi-anoxic zones in aquatic environments. The magnetosensing property is enabled by the magnetosomes, sub-cellular organelles each composed of nanometer-sized iron-based magnetic particle (magnetite or gregite) enclosed in a protein-rich lipid membrane. The magnetite exhibits high chemical purity, with only neglected incorporation of trace element compared to iron, as compared to its abiotic synthesis. In each bacterium, the magnetosomes are arranged in a chain-like fashion which creates the dipole moment needed for magnetic-reception ${ }^{17-20}$. MamM is a CDF iron transporter found in the magnetosome membrane that facilitates the accumulation of iron inside the magnetosomes, thus enabling the synthesis of the magnetic particles. It has been previously shown that the deletion of mam $M$ gene, or even only of its CTD, abolishes magnetic particle formation ${ }^{10}$. Furthermore, mutations in its metal binding sites in both the TMD and CTD cause defective formation of the magnetic particles in vivo ${ }^{10,14}$. MamM CTD was well characterized in vitro: its crystal structure revealed that it has the characteristic fold of CDF proteins; the CTD structure was shown to be crucial for the overall protein function; DDMC binding to the CTD causes a conformational change from a dynamic-apo form to a rigid, more closed V-shaped structure; and it binds different DDMCs distinctively ${ }^{14,16,21,22}$. Comprehensive biophysical analysis of MamM CTD in the presence of $\mathrm{Zn}^{2+}$ and $\mathrm{Fe}^{2+}$ and in vivo studies showed that MamM CTD dimer binds three ions by two binding sites: a central binding site composed of D249 and $\mathrm{H} 285$ from both monomers, and two symmetrical peripheral binding sites, each composed of $\mathrm{H} 264$ from one monomer and E289 from the second monomer ${ }^{14,22}$ (Figure S1). The crystal structures of MamM CTD bound to $\mathrm{Cu}^{2+}, \mathrm{Ni}^{2+}$ and $\mathrm{Cd}^{2+}$ were previously determined, confirming the participation of the binding site residues in the chelation of the metals ${ }^{16}$. The $\mathrm{Cu}^{2+}$-bound structure shows tighter conformation compared to the apo form, with copper ions bound at the two sites by H285, H264 and E289 (through a water molecule). The Cd ${ }^{2+}$-bound and $\mathrm{Ni}^{2+}$-bound structures showed no conformational changes compared to the apo form; however, $\mathrm{Cd}^{2+}$ is bound by $\mathrm{D} 249$ and $\mathrm{H} 285$ in the central site and $\mathrm{Ni}^{2+}$ by $\mathrm{H} 264$. Complementary biophysical studies in solution showed that $\mathrm{Ni}^{2+}, \mathrm{Cu}^{2+}, \mathrm{Zn}^{2+}$ and $\mathrm{Cd}^{2+}$ bind differently to the protein in terms of number of binding sites and affinity, and that the binding of all these metals leads to the same tight conformation is solution as the binding of $\mathrm{Fe}^{2+}$. These studies also showed that $\mathrm{Mn}^{2+}$ does not bind the CTD, all implying that the CTD has a direct role in metal selectivity ${ }^{16}$. MamM is a well characterized protein, in terms of its function, structure and mechanism. Furthermore, changing MamM metal selectivity so to insert different metals into the magnetosomes and enable the synthesis of chemically-modified magnetic particles in MTB, can have diverse biotechnological application ${ }^{23}$. Hence, MamM can serve as an effective model to study the relationship between CDFs' CTDs metal binding site composition and structure.

Willing to influence the ability to bind non-native DDMCs to the CTD of MamM, here we investigated the impact of MamM CTD metal binding site composition on the CTD responsivity to different DDMCs, in terms of its binding ability and its related conformational changes. For that, we designed nine new mutated constructs of MamM CTD (residues 215-318), where we altered the binding site residues to different residues that tend to bind DDMCs with high propensities ${ }^{4}$. We created the D249H and H285D mutations to investigate whether a central binding site composed of four histidines (uncharged bulky binding site) or four aspartates (highly charged binding site) can maintain the DDMCs binding abilities. Similarly, we created the H264E and E289H mutations for the investigation of the peripheral sites. To understand the importance of the location of the residues within the binding site for DDMCs binding, we also created two double mutations, D249H-H285D and H264E-E289H. Lastly, to examine the alkyl group length and the charge importance and influence on binding, we created the D249N, D249E and E289D mutations. All mutant constructs were studied in the presence of different DDMCs using X-ray crystallography and Trp-fluorescence spectrometry. The Trp-fluorescence spectrometry results reveal that each mutation has a different effect on the ability to bind the DDMCs in solution. The crystal structures of 22 mutants/mutant-DDMC pairs were detected and compared to the previously solved structures of MamM CTD with and without DDMCs, showing a range of possible conformations and binding sites that depend on both the nature of the substitution and the DDMC identity. Overall, our results show that the CDFs CTD metal binding sites might be altered to change the response of this domain to different DDMCs in vitro, and to impact the regulation of the whole protein. This study enables a rational design of MamM mutants so as to change its metal selectivity and to control its regulation, and this would constitute a first step for a future synthesis of magnetic particles with different chemical properties in vivo.

\section{Results and discussion}

All nine designed mutants were well expressed and purified and were characterized in the presence of different DDMCs using X-ray crystallography and Trp-fluorescence spectrometry. To achieve better understanding on the conformational changes that occur due to DDMC binding in the different MamM CTD mutants, we attempted to crystallize MamM CTD mutants with diverse DDMCs $\left(\mathrm{Fe}^{2+}, \mathrm{Mn}^{2+}, \mathrm{Ni}^{2+}, \mathrm{Cu}^{2+}, \mathrm{Zn}^{2+}\right.$ and $\left.\mathrm{Cd}^{2+}\right)$ under various conditions. We were able to successfully solve 22 structures of different MamM CTD mutants/MamM CTD mutant-DDMC pairs, but not with iron (Table 1; see Tables S1-S3 for more crystallographic details). Among these structures, we detected the structures of all mutants; some in the presence of DDMCs, some in their apo structures without noticeable metal bound and some in both states. We further characterized the differences in the binding abilities of all nine mutants and of the three binding-site deletion mutants: MamM CTD D249A-H285A (deletion of the central binding site), H264A-E289A (deletion of the peripheral binding site) and D249A-H264A (deletion of both sites) using Trp-fluorescence spectrometry, as previously described ${ }^{14,16,22}$. Here we titrated nonnative DDMCs that were previously shown to be transported by other varied CDF proteins $\left(\mathrm{Cd}^{2+}, \mathrm{Mn}^{2+}, \mathrm{Ni}^{2+}\right.$, $\mathrm{Cu}^{2+}$ and $\mathrm{Zn}^{2+}$ ) into MamM CTD mutant solutions and measured the concentration-dependent changes in the tryptophan emission spectra (see Methods for analysis description and Figure S1; as metal binding to MamM CTD requires a basic $\mathrm{pH}^{14,22}$, and as titration of $\mathrm{Fe}^{2+}$ leads to iron precipitation and inaccurate results in such $\mathrm{pH}$, 


\begin{tabular}{|l|l|l|l|l|l|l|}
\hline MamM CTD form & $\mathbf{Z n}^{2+}$ & $\mathbf{C d}^{2+}$ & $\mathbf{M n}^{2+}$ & $\mathbf{C u}^{2+}$ & $\mathbf{N i}^{2+}$ & $\mathbf{F e}^{2+}$ \\
\hline D249E & $6 \mathrm{H} 5 \mathrm{~K}^{\mathrm{a}}$ & $6 \mathrm{H} 9 \mathrm{Q}$ & & & & \\
\hline $\mathrm{D} 249 \mathrm{H}$ & & $6 \mathrm{H} 84$ & & $6 \mathrm{H} 83$ & & \\
\hline $\mathrm{D} 249 \mathrm{~N}$ & $6 \mathrm{H} 87$ & $6 \mathrm{H} 8 \mathrm{~A}$ & $6 \mathrm{H} 88^{\mathrm{b}}$ & $6 \mathrm{H} 89$ & $6 \mathrm{H} 8 \mathrm{D}$ & \\
\hline $\mathrm{H} 285 \mathrm{D}$ & & $6 \mathrm{H} 9 \mathrm{~T}$ & $6 \mathrm{H} 8 \mathrm{I}^{\mathrm{b}}$ & & & \\
\hline D249H-H285D & & & $6 \mathrm{HA} 2^{\mathrm{b}}$ & & & \\
\hline E289D & & $6 \mathrm{HHS}$ & $6 \mathrm{H} 9 \mathrm{P}$ & & & \\
\hline E289H & & & & & $6 \mathrm{H} 81$ & \\
\hline $\mathrm{H} 264 \mathrm{E}$ & $6 \mathrm{H} 5 \mathrm{M}, 6 \mathrm{H} 5 \mathrm{U}$ & $6 \mathrm{H} 8 \mathrm{G}, 6 \mathrm{HAO}$ & $6 \mathrm{H} 5 \mathrm{~V}^{\mathrm{b}}$ & & & \\
\hline $\mathrm{H} 264 \mathrm{E}-\mathrm{E} 289 \mathrm{H}$ & & $6 \mathrm{H} 85$ & $6 \mathrm{HAN}$ & & & \\
\hline
\end{tabular}

Table 1. List of MamM CTD mutant-DDMC pairs with crystal structures detected in this study. ${ }^{a}$ All existing structures are indicated by their pdb codes. ${ }^{b}$ All MamM CTD mutants that were crystallized in the presence of $\mathrm{Mn}^{2+}$, however $\mathrm{Mn}^{2+}$ was not detected in the electron density map hence the structures are referred as apo structures.
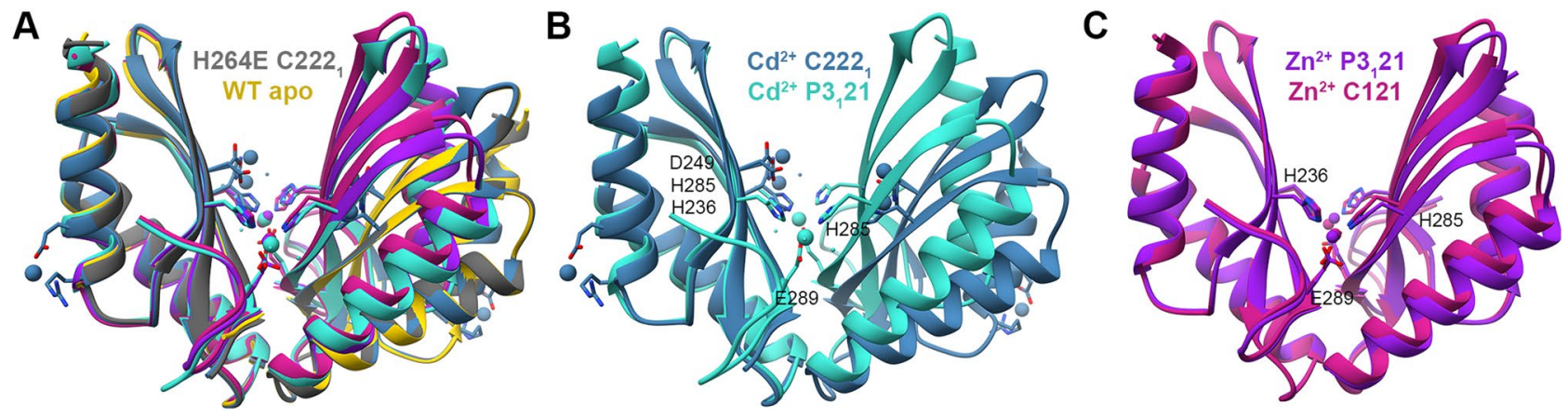

Figure 1. Crystal structures of MamM CTD H264E mutant with different metals. (A) Crystal structures of MamM CTD H264E with no metal bound $\left(\mathrm{Mn}^{2+}\right.$ in crystallization condition, dim gray, pdb code: $\left.6 \mathrm{H} 5 \mathrm{~V}\right)$, with $\mathrm{Cd}^{2+}$ (SG P3 21 , turquoise, pdb code:6HAO; SG C222 ${ }_{1}$, steel blue, pdb code: $6 \mathrm{H} 8 \mathrm{G}$ ) and with $\mathrm{Zn}^{2+}$ (SG P3 21 , purple, pdb code:6H5M; SG C121, violet red, pdb code:6H5U) overlaid onto apo MamM CTD WT structure (SG C2221, gold, pdb code:3W5X ${ }^{14}$ ). (B) Crystal structures of MamM CTD H264E with $\mathrm{Cd}^{2+}$ at two different SGs ( P $_{1} 21$ turquoise, $\mathrm{C} 222_{1}$ steel blue) show different binding sites and conformations of the mutant with $\mathrm{Cd}^{2+}$. (C) Crystal structures of MamM CTD H264E with $\mathrm{Zn}^{2+}$ at two different SGs (P3 21 purple, C121 violet red) show the same $\mathrm{Zn}^{2+}$ binding site which involves residues from both the central and peripheral binding sites, and the same closed conformation. All MamM CTD construct structures were overlaid and all structural figures were prepared using UCSF Chimera package, version $1.12^{37}$.

even under inert conditions ${ }^{16}$, iron was not retested). Below, we discuss the main differences observed between peripheral site mutants with different DDMCs, central site mutants with different DDMCs, or between different mutants with the same DDMC, while considering all related results.

Impact of peripheral site mutants on DDMC binding. The peripheral site mutants in which we were able to determine their X-ray structure in the presence of different DDMCs are MamM CTD H264E and MamM CTD E289D, hence only these mutants will be analyzed and discussed in this section.

MamM CTD H264E with different metals. We solved the crystal structures of MamM CTD H264E with $\mathrm{ZnCl}_{2}$ in two different space groups, with $\mathrm{CdCl}_{2}$ in two different space groups and in the presence of $\mathrm{MnCl}_{2}$ in the crystallization condition-without, however, detecting $\mathrm{Mn}^{2+}$ in the electron density map (and therefore we relate to this structure as the apo structure) (Fig. 1A). The $\mathrm{Cd}^{2+}$-bound structures exhibit two conformations. The first structure [space group (SG) C222 $2_{1}$ exhibits the same conformation as the wildtype (WT) apo and H264E apo but with two $\mathrm{Cd}^{2+}$ ions bound by each monomer's D249 and H285 residues (Fig. 1A,B). However, the occupancy of each ion in the electron density map is half, suggesting that only one ion can be bound by each monomer in either of the two binding sites. Another $\mathrm{Cd}^{2+}$ ion was detected in the periphery of each monomer, bound in a way that seems to have no influence on the protein conformation (Fig. 1B). The second structure (SG P $3_{1} 21$ ) exhibits much tighter dimerization compared to that of the apo forms, with two symmetrical binding sites that involve $\mathrm{H} 285$ from one monomer and H236 and E289 from the second monomer. In none of the structures does the E264 residue participate in the $\mathrm{Cd}^{2+}$ binding. Both $\mathrm{Zn}^{2+}$-bound structures (SGs C121 and P3 21) exhibit the same conformation and binding sites which are shared with the second $\mathrm{Cd}^{2+}$-bound structure (Fig. $\left.1 \mathrm{~A}, \mathrm{C}\right)$, suggesting that this might be a preferred conformation for both cations. Surprisingly, the H264E- $\mathrm{Zn}^{2+}$ closed conformation structures were not observed for the WT form (diffracting crystals for the WT CTD with $\mathrm{Zn}^{2+}$ 
were not obtained), which may be a result of the different charge of the binding site. Therefore, we also compared them using Trp-fluorescence spectrometry.

H264E-Cd ${ }^{2+}$ Trp-fluorescence scans show much smaller blue shift compared to the WT protein (and the smallest among all mutants; Fig. 2B, upper panel), while H264E- $\mathrm{Zn}^{2+}$ Trp-fluorescence scans show some smaller blue shift compared to the WT protein (Fig. 2A, upper panel), yet much larger compared to that of $\mathrm{Cd}^{2+}$. Nevertheless, previous PELDOR results of the WT CTD with $\mathrm{Zn}^{2+}$ and $\mathrm{Cd}^{2+}$-where $\mathrm{C} 275$ was spin-labeled in the WT CTD, and the distances between the two labeled position of the dimer in the apo and metal-bound states were measured-exhibit similar spin-labeling distances for both DDMCs, whereas the $\mathrm{Cd}^{2+}$-bound protein exhibits smaller blue-shift as compared to $\mathrm{Zn}^{2+}$, suggesting that $\mathrm{Cd}^{2+}$ causes smaller blue-shift although it undergoes similar closure in the presence of both DDMCs ${ }^{16}$.

The calculated PELDOR spin-label distance of the apo WT protein is $\sim 7 \AA$ longer than the experimentally determined WT $\mathrm{Zn}^{2+}$-bound distance ${ }^{22}$. This difference in the spin-label distances was also shown for the apo and $\mathrm{Cu}^{2+}$-bound WT, both in solution and crystal forms, suggesting that the WT binds $\mathrm{Zn}^{2+}$ and $\mathrm{Cu}^{2+}$ similarly in solution and that the $\mathrm{Cu}^{2+}$-bound crystal structure represents also the conformation of the $\mathrm{Cu}^{2+}$ - and $\mathrm{Zn}^{2+}$-bound $\mathrm{WT}$ in solution ${ }^{16}$. However, the difference in distance between the same spin-labelled cysteine residues in the $\mathrm{H} 264 \mathrm{E}-\mathrm{Zn}^{2+}$ and the WT apo form is only $\sim 3 \AA$, which indicates a smaller conformational change as compared to that in the WT protein. Comparison of the $\mathrm{Cu}^{2+}$-bound WT structure (which represents the $\mathrm{Zn}^{2+}$-bound WT as well) to the $\mathrm{H} 264 \mathrm{E}-\mathrm{Zn}^{2+}$ bound structures reveals different orientation of the monomers, with the $\mathrm{Cu}^{2+}$-bound WT structure exhibiting much closer tryptophan residues with different environment as compared to the H264E$\mathrm{Zn}^{2+}$ bound structures. Taking together all these crystallographic and fluorometric data, these results indicate that: (1) The addition of $\mathrm{Cd}^{2+}$ to H264E causes a minor conformational change in solution, if at all, and (2) Different conformational change occurs in H264E in the presence of $\mathrm{Zn}^{2+}$ compared to that in the WT, with the crystal structure probably representing also the conformation in solution.

MamM CTD E289D with different metals. We solved the crystal structures of MamM CTD E289D with CdCl and $\mathrm{MnCl}_{2}$ (Fig. 3A). The E289D- $\mathrm{Mn}^{2+}$-bound structure contains three monomers in the asymmetric unit: two of them compose one biological dimer, and the third composes a biological assembly together with a monomer from an adjacent unit. Since a weak $\mathrm{Mn}^{2+}$ density was detected only in the latter dimer-bound to $\mathrm{H} 264$ and a water molecule-and as both dimers possess similar conformation (Fig. 3B), it appears that the $\mathrm{Mn}^{2+}$ is bound non-specifically at that position. The conformation of this structure is much tighter than that of the WT apo form. In this structure, the D289 residue from one monomer forms a hydrogen bond with the second monomer $\mathrm{H} 264$, which stabilizes the closed conformation. In the WT protein the E289 residue could form this hydrogen bond, but as the glutamate residue is longer, the distance and angles between the other protomer's residues would be different, which would lead to a smaller, less-stabilized network of bonds not sufficient for a steady closed conformation. This mutant is a great example of the dynamic conformation of the CTD in its unbound state, and of how the CTD conformation can be shifted by a seemingly minor mutation. The E289D-Cd ${ }^{2+}-$ bound structure contains seven monomers in the asymmetric unit; six of them compose three biological dimers, and the seventh composes a biological assembly together with a monomer from an adjacent unit (Fig. 3C-E). All four dimers exhibit the same $\mathrm{Cd}^{2+}$ binding sites and conformation, which is tighter than that of the WT apo form but less tight than that of the E289D-Mn' $\mathrm{Mn}^{2+}$-bound structure (Fig. 3A,C). The dimer organization in the crystal (Fig. 3D) reveals a unique $\mathrm{Cd}^{2+}$-pair binding site that involves residues from both dimer monomers and from a third monomer (that by itself form a dimer) (Fig. 3E,F). The binding site involves residues from both central and peripheral binding sites; this binding site, and hence the conformation, could not exist in the presence of the longer E289 residue in the WT protein as it would deform the specific binding site geometry, showing again how this seemingly non-disruptive mutation can lead to different binding abilities. As this structure is not biological, it would not extend beyond this discussion.

The Trp-fluorescence maximum emission wavelength of E289D without the presence of any metals is shorter than that of the WT (Figure S3), although the residue is distant from W247 and therefore should not directly impact the fluorescence signal. This suggests that the initial conformation of the protein is tighter in this mutant form, as the E289D-Mn ${ }^{2+}$ structure also implies. The addition of $\mathrm{Zn}^{2+}$ and $\mathrm{Cd}^{2+}$ show some smaller blue-shift compared to the WT, while $\mathrm{Ni}^{2+}$ and $\mathrm{Cu}^{2+}$ show very similar trends in the signal quenching compared to the WT (Fig. 2). However, as the initial maximum wavelength of E289D is shorter, it is not surprising to detect smaller shift in the wavelength; the E289D- $\mathrm{Zn}^{2+}$ and WT- $\mathrm{Zn}^{2+}$ maximum emission wavelengths at saturation are the same, hinting at a similar conformation, while that of E289D-Cd ${ }^{2+}$ is shorter than the WT-Cd ${ }^{2+}$ maximum emission wavelength at saturation, implying an even tighter conformation in this mutant form. For all other metals, as the signal quenching corresponds to that of the WT, one can expect similar conformations.

Impact of central site mutants on DDMC binding. The central site mutants in which we were able to determine their X-ray structure in the presence of different DDMCs are MamM CTD D249N, MamM CTD D249H and MamM CTD D249E. The crystal structures of MamM CTD D249E with $\mathrm{CdCl}_{2}$ and $\mathrm{ZnCl}_{2}$ and of MamM CTD D249H with $\mathrm{CdCl}_{2}$ and $\mathrm{CuSO}_{4}$ were solved, and their conformations and binding sites can be observed in Figure S4. As they have no unique conformations compared to the structures that are discussed in this section, they will be discussed only in the metal-based analysis below, therefore only the D249N mutant will be analyzed and discussed in this section.

MamM CTD D249N with different metals. We solved the crystal structures of MamM CTD D249N with $\mathrm{ZnCl}_{2}$, $\mathrm{CdCl}_{2}, \mathrm{NiCl}_{2}$ and $\mathrm{CuSO}_{4}$ and in the presence of $\mathrm{MnCl}_{2}$ in the crystallization condition, however without detecting $\mathrm{Mn}^{2+}$ in the electron density map (and therefore we relate to this structure as the apo structure) (Fig. 4A). 

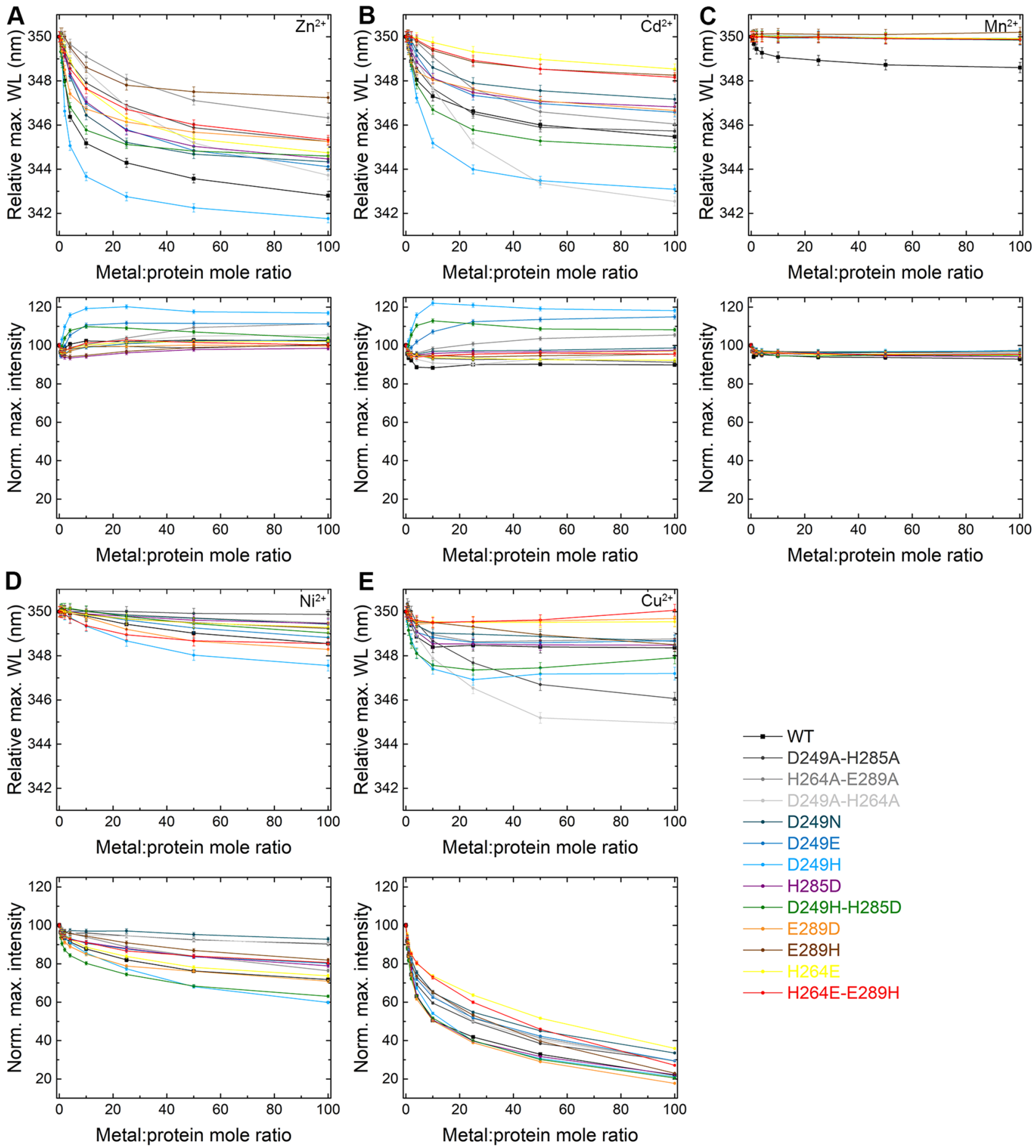

Figure 2. Fluorescence scans of MamM CTD constructs with varying metal concentrations. For (A-E) panels, the upper graph represents the maximum wavelength (normalized to $350 \mathrm{~nm}$ for easier comparison) as function of metal:protein ratio, while the lower panel represents the normalized fluorescence intensity compared to no metal as function of metal:protein ratio, of all MamM CTD constructs (WT, black, adapted from Barber-Zucker et al. ${ }^{16}$; D249A-H285A, dark gray; H264A-E289A, gray; D249A-H264A, light gray; D249N, turquoise; D249E, dark blue; D249H, light blue; H285D, purple; D249H-H285D, green; E289D, orange; E289H, brown; H264E, yellow; H264E-E289H, red). MamM CTD proteins at $5 \mu \mathrm{M}$ concentration were titrated using metal solutions: (A) $\mathrm{Zn}^{2+}$, (B) $\mathrm{Cd}^{2+}$, (C) $\mathrm{Mn}^{2+}$, (D) $\mathrm{Ni}^{2+}$, and (E) $\mathrm{Cu}^{2+}$, to reach different metal:protein ratios (intensity was normalized due to the change in MamM CTD concentration) and emission spectra were recorded. Samples were measured at an excitation of $\lambda$ ex $297 \mathrm{~nm}$ and the emission spectrum for each metal concentration was recorded between $310-450 \mathrm{~nm}$. For each protein, the data presented is the average of three independent measurements. See also Figures S2-S3 for easier interpretation of the data. 
A

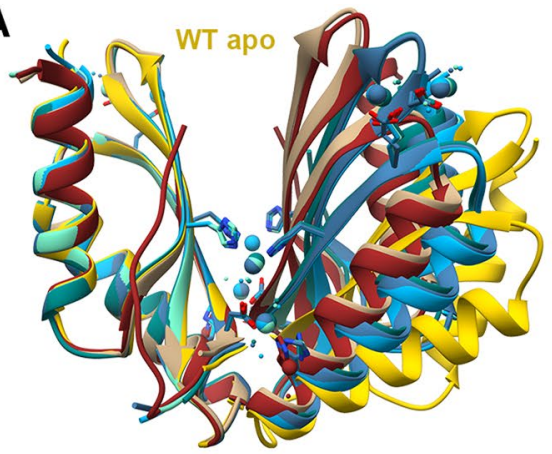

D

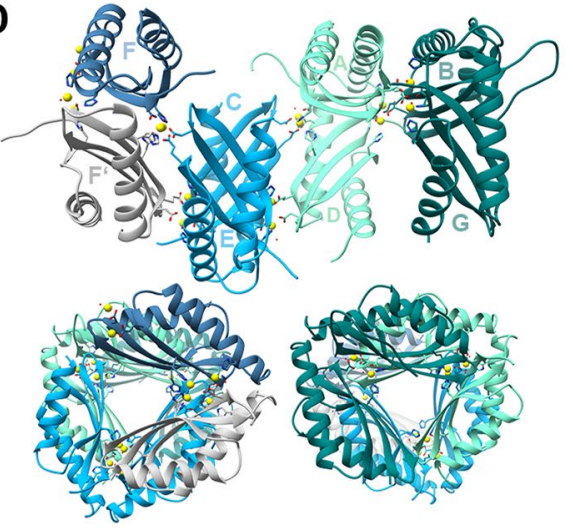

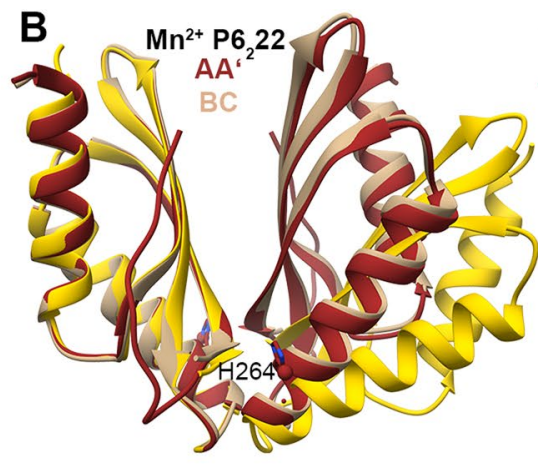

$E$

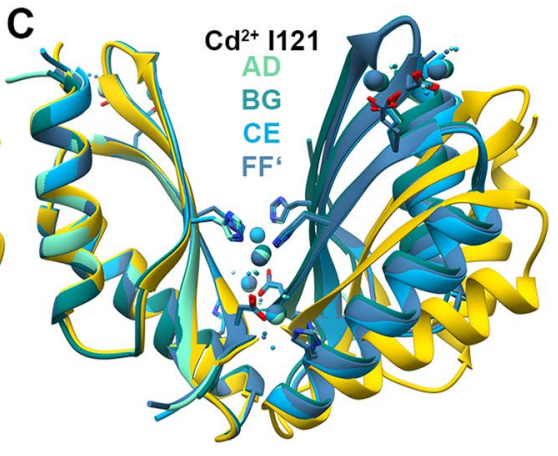

$\mathbf{F}$

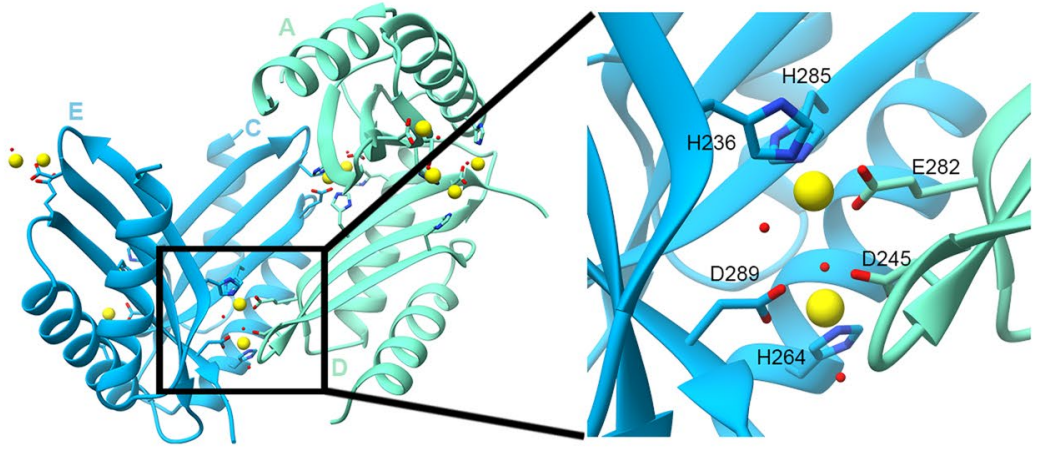

Figure 3. Crystal structures of MamM CTD E289D mutant with different metals. (A) Crystal structures of MamM CTD E289D with Cd ${ }^{2+}$ (SG I121, blue shades, pdb code: $6 \mathrm{HHS}$ ) and $\mathrm{Mn}^{2+}$ (SG P6 22 , brown shades, pdb code: $6 \mathrm{H} 9 \mathrm{P}$ ) overlaid onto apo MamM CTD WT structure (SG C222 ${ }_{1}$, gold, pdb code: $3 \mathrm{~W} 5 \mathrm{X}^{14}$ ). All possible dimers are shown: four for the $\mathrm{E} 289 \mathrm{D}-\mathrm{Cd}^{2+}$ formed from seven monomers from the asymmetric unit and a monomer from an adjacent unit, and two for E289D- $\mathrm{Mn}^{2+}$, formed from three monomers from the asymmetric unit and a monomer from an adjacent unit. (B) Crystal structure of MamM CTD E289D with $\mathrm{Mn}^{2+}$ (chains $\mathrm{A}+\mathrm{A}^{\prime}$ (tag for a monomer from an adjacent unit) in brown and chains $\mathrm{B}+\mathrm{C}$ in tan) exhibits tighter conformation compared to the apo MamM CTD WT (gold). $\mathrm{Mn}^{2+}$ is bound by $\mathrm{H} 264$ and water molecule in each monomer of only the A+ $\mathrm{A}^{\prime}$ dimer. (C) Crystal structure of MamM CTD E289D with $\mathrm{Cd}^{2+}$ (chains A+D in aquamarine, chains $\mathrm{B}+\mathrm{G}$ in dark cyan, chains $\mathrm{C}+\mathrm{E}$ in deep sky blue and chains $\mathrm{F}+\mathrm{F}^{\prime}$ in steel blue) exhibits tighter conformation compared to the apo MamM CTD WT (gold), and different than that of E289D-Mn ${ }^{2+}$. Every $\mathrm{Cd}^{2+}$-pair is chelated by residues from three monomers (see Panels $\mathrm{E}+\mathrm{F}$ ); the upper $\mathrm{Cd}^{2+}$ pairs are shown for clarity to demonstrate how each monomer chelates the $\mathrm{Cd}^{2+}$ ions by three different sites. (D) Crystal packing of MamM CTD E289D with $\mathrm{Cd}^{2+}$ from three different angles. The asymmetric unit contains seven monomers that compose three biological dimers (chains $\mathrm{A}+\mathrm{D}$ in aquamarine, chains $\mathrm{B}+\mathrm{G}$ in dark cyan and chains $\mathrm{C}+\mathrm{E}$ in deep sky blue). The seventh chain comprises an identical dimer with a monomer from an adjacent unit (chain $\mathrm{F}$ in steel blue and chain $\mathrm{F}^{\prime}$ from the adjacent unit in gray). (E) One dimer (chains $\mathrm{C}+\mathrm{E} /$ chains $\mathrm{A}+\mathrm{D}$ ) and one monomer (chain D/chain $\mathrm{C}$, respectively) are involved in the chelation of each $\mathrm{Cd}^{2+}$-pair. $(\mathbf{F})$ Magnification of the $\mathrm{Cd}^{2+}$-pair binding site (Panel E, involving the chains $\mathrm{C}+\mathrm{D}+\mathrm{E}$ ): $\mathrm{H} 236$ and D289 from chain E, H285 and H264 from chain C, D245 and E282 from chain D, and three water molecules, participate in the chelation of the $\mathrm{Cd}^{2+}$-pair.

The $\mathrm{Zn}^{2+}$-bound structure exhibits the same tighter conformation as the $\mathrm{H} 264 \mathrm{E}-\mathrm{Zn}^{2+}$ structure; nonetheless, it contains three symmetrical pairs of $\mathrm{Zn}^{2+}$ binding sites (Fig. 4B). One of the binding sites is shared with the $\mathrm{H} 264 \mathrm{E}-\mathrm{Zn}^{2+}$ structure, while $\mathrm{H} 264$ residue binds the additional two $\mathrm{Zn}^{2+}$ ions in the periphery of each monomer, suggesting that the $\mathrm{H} 264$ residue has a role in attracting $\mathrm{Zn}^{2+}$ ions to the $\mathrm{CTD}$. The $\mathrm{Cd}^{2+}, \mathrm{Ni}^{2+}$ and $\mathrm{Cu}^{2+}$ bound structures have the same conformation as the WT apo form (Fig. 4C). While $\mathrm{Ni}^{2+}$ and $\mathrm{Cu}^{2+}$ are bound solely by $\mathrm{H} 264$ in each monomer (in addition to water molecules), one $\mathrm{Cd}^{2+}$ is chelated by $\mathrm{H} 285$ in each monomer, by water molecules and by $\mathrm{H} 213$ and E215 from a non-biological monomer from an adjacent unit. This implies that in contrast to the aspartate residue at position 249 , asparagine cannot bind $\mathrm{Cd}^{2+}$. The last structure, that of the unbound D249N mutant, shows a very tight conformation compared to the WT apo and the D249N- $\mathrm{Zn}^{2+}$ bound structures (Fig. 4A,D). This conformation is enabled by the D249N substitution that abolishes the original negative charge from the central binding site-which causes repulsion between the two monomers-and instead adds possible hydrogen bonds between the two asparagine residues that stabilize the closely-contacted monomers. Overall, the D249N crystal structures exhibit versatile conformation modes and binding sites that depend on the DDMC identity.

Trp-fluorescence results (Fig. 2, S2 and S3), mainly of $\mathrm{Ni}^{2+}$ and $\mathrm{Cd}^{2+}$ but also of $\mathrm{Cu}^{2+}$ and $\mathrm{Zn}^{2+}$ show smaller spectral shift (quenching/blue shift) when titrated to D249N, as compared to that of the WT. This means that 

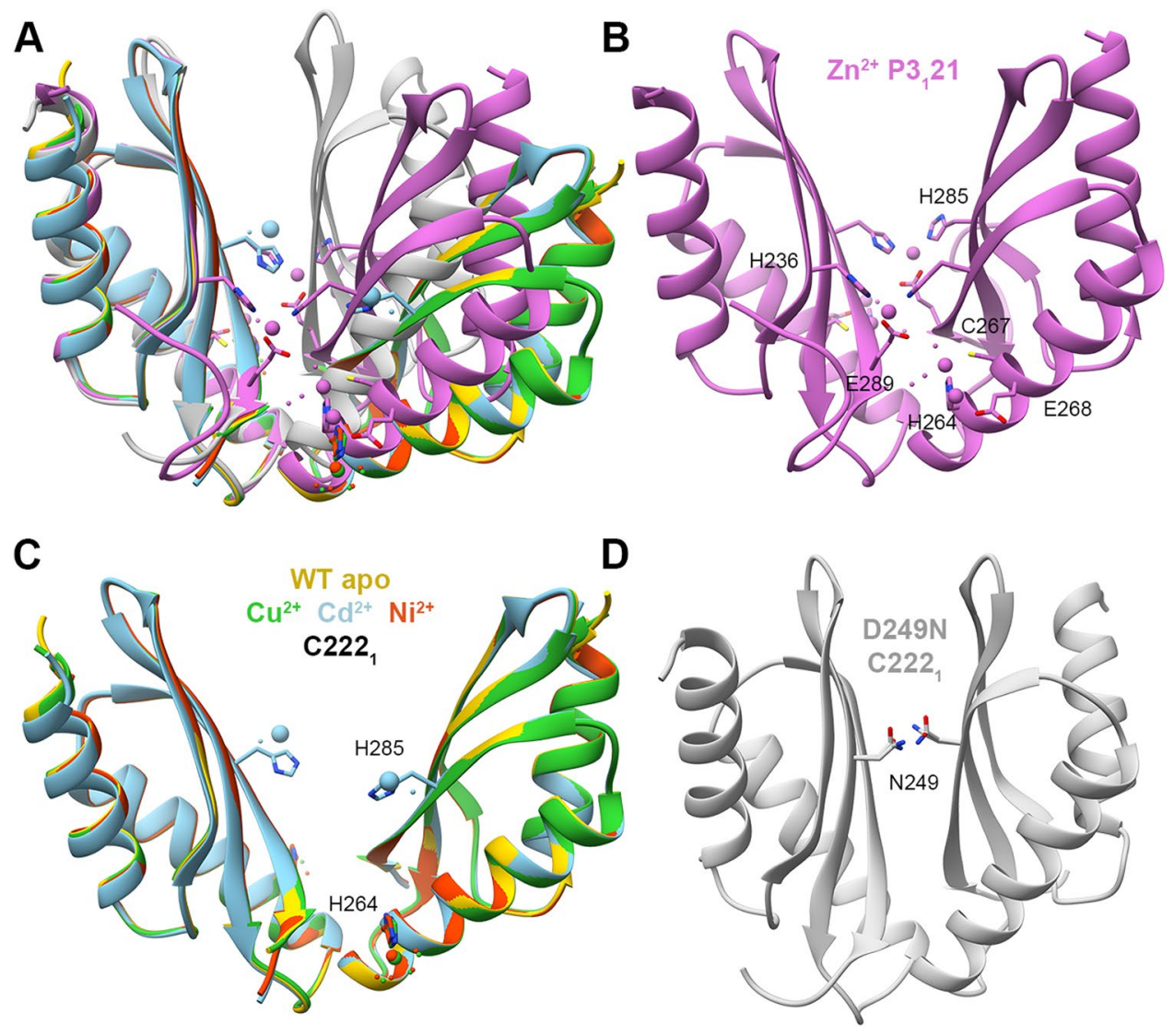

Figure 4. Crystal structures of MamM CTD D249N mutant with different metals. (A) Crystal structures of MamM CTD D249N with no metal bound $\left(\mathrm{Mn}^{2+}\right.$ in crystallization condition, light gray, pdb code: $\left.6 \mathrm{H} 88\right)$, with $\mathrm{Cd}^{2+}$ (SG C222, sky blue, pdb code: $6 \mathrm{H} 8 \mathrm{~A}$ ), $\mathrm{Cu}^{2+}$ (SG C222, lime green, pdb code: $6 \mathrm{H} 89$ ), $\mathrm{Ni}^{2+}$ (SG C222 ${ }_{1}$, orange red, pdb code: $6 \mathrm{H} 8 \mathrm{D}$ ) and $\mathrm{Zn}^{2+}$ (SG P3 21 , orchid, pdb code: 6H87), overlaid onto apo MamM CTD WT structure (SG C222 1 , gold, pdb code: $3 \mathrm{~W}^{2} \mathrm{X}^{14}$ ). (B) Crystal structure of MamM CTD D249N with Zn ${ }^{2+}$ exhibits the same $\mathrm{Zn}^{2+}$ binding site as that of $\mathrm{H} 264 \mathrm{E}-\mathrm{Zn}^{2+}$ and the same conformation, with another two $\mathrm{Zn}^{2+}$ ions per monomer bound in two sites that involve H264. (C) Crystal structures of MamM CTD D249N with Cd ${ }^{2+}$ (sky blue), $\mathrm{Cu}^{2+}$ (lime green) and $\mathrm{Ni}^{2+}$ (orange red) show the same conformation as apo MamM CTD WT (gold). While $\mathrm{Cu}^{2+}$ and $\mathrm{Ni}^{2+}$ are chelated by $\mathrm{H} 264$ and water molecules, $\mathrm{Cd}^{2+}$ is bound by $\mathrm{H} 285$, water molecules, and H213 and E215 from a non-biological monomer from an adjacent unit (not shown). (D) Crystal structures of MamM CTD D249N with no metal bound $\left(\mathrm{Mn}^{2+}\right.$ in crystallization condition) show very tight dimeric conformation that is stabilized by hydrogen bonds between the N249 residues from both monomers, which are replacing the charged and repulsive native-D249 in this mutant construct.

$\mathrm{Ni}^{2+}$ and $\mathrm{Cd}^{2+}$ are not bound to the $\mathrm{D} 249 \mathrm{~N}$ mutant in a way that causes spectral shifts (in close proximity to the W247, and for $\mathrm{Cd}^{2+}$ also in a way that leads to conformational change). This is further supported by the crystal structures-where the $\mathrm{Ni}^{2+}$ is bound only in the periphery of the protein (by one residue which is distant from W247), and the $\mathrm{Cd}^{2+}$ is chelated only by $\mathrm{H} 285$ which is more distant than the D249 in the bound WT form ${ }^{16}$-and where no conformational changes are shown. Similarly to H264E- $\mathrm{Zn}^{2+}$, that also exhibits the same crystallographic conformation, D249N-Zn ${ }^{2+}$ shows smaller blue-shift compared to WT and different binding site composition from $\mathrm{WT}^{22}$, indicating that it adopts the same conformation in solution as the crystal structure.

DDMC binding to different mutants. For $\mathrm{Zn}^{2+}, \mathrm{Cd}^{2+}, \mathrm{Ni}^{2+}$ and $\mathrm{Cu}^{2+}$, we were able to determine the crystal structure of several mutants bound to each of these DDMCs. Here, we focus on the analyses of the differences between the ability of different mutants to bind $\mathrm{Zn}^{2+}, \mathrm{Ni}^{2+}$ and $\mathrm{Mn}^{2+}$. Detailed analyses of $\mathrm{Cd}^{2+}$ and $\mathrm{Cu}^{2+}$ binding to the varied mutants is given in the Supplementary Results and Discussion section.

MamM CTD mutants binding to $\mathrm{Zn}^{2+}$. We solved the crystal structures of MamM CTD H264E (two different SGs), D249E and D249N with $\mathrm{Zn}^{2+}$. All structures exhibit the same fold and conformation, tighter than that of the WT apo (Fig. 5A) and different from the $\mathrm{Cu}^{2+}$-bound WT structure, which is assumed to represent the $\mathrm{Zn}^{2+}$-bound structure as well due to similarities shown in previous biophysical analyses ${ }^{16}$. Examination of the $\mathrm{Zn}^{2+}$ binding sites reveals the importance of the $\mathrm{H} 264$ residue for $\mathrm{Zn}^{2+}$ binding. All three mutants have a shared symmetrical binding site, involving H285 from one monomer and H236 and E289 from the second monomer 


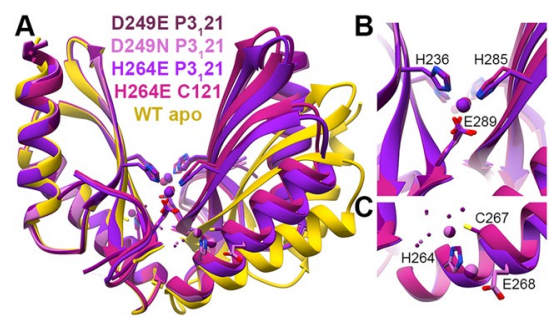

Figure 5. Crystal structures of different MamM CTD constructs bound to $\mathrm{Zn}^{2+}$. (A) Crystal structures of MamM CTD D249E with Zn ${ }^{2+}$ (SG P3 121 , dark magenta, pdb code: 6H5K), MamM CTD D249N with Zn ${ }^{2+}$ (SG P3 21, orchid, pdb code: $6 \mathrm{H} 87$ ) and MamM CTD H264E with $\mathrm{Zn}^{2+}$ (SG P3 1 21, purple, pdb code: 6H5M; SG C121, violet red, pdb code: $6 \mathrm{H} 5 \mathrm{U}$ ) overlaid onto apo MamM CTD WT structure (SG C2221, gold, pdb code: $3 \mathrm{~W} 5 \mathrm{X}^{14}$ ). (B) The main $\mathrm{Zn}^{2+}$ binding site that is shared to all MamM CTD $\mathrm{Zn}^{2+}$-bound structures involves H236 and E289 from one monomer and $\mathrm{H} 285$ from the second monomer. (C) The peripheral binding site that is found only in MamM CTD D249E and D249N structures involves water molecules and residues only from one monomer (symmetric site): C267, E268 and H264. As can be evident in the H264E structures, the substitution of $\mathrm{H} 264$ to glutamate abolishes this site.

(Fig. 5B). This binding site, that involves residues from both the central and peripheral binding sites, does not involve any of the mutated residues. The $\mathrm{Zn}^{2+}$-bound structure could not be obtained for the WT form. However, previous results suggest that the $\mathrm{Zn}^{2+}$ and $\mathrm{Cu}^{2+}$ lead to the same conformation in solution and have the same binding sites ${ }^{16,22}$, suggesting that this site is not natural. The D249E and D249N mutants contain a second symmetrical binding site. In this site, the $\mathrm{H} 264$ residue chelates two $\mathrm{Zn}^{2+}$ ions together with $\mathrm{C} 267$ and $\mathrm{E} 268$ from the same monomer and water molecules (Fig. 5C). This binding site seems not to influence the protein conformation, as it involves residues only from one monomer and as the H264E mutant shows the same fold. Nevertheless, the ability to bind four additional $\mathrm{Zn}^{2+}$ ions per dimer by $\mathrm{H} 264$ residue suggests that it might have a role in the attraction of ions to the CTD as an intermediate binding site, before the cations can be transported from this site to the second CTD binding site or to the TMD transport site. When comparing the Trp-fluorescence results of the WT and these mutants (Fig. 2A, top panel), all three mutants show similar blue shift somewhat smaller than that of the WT, with the H264E showing the smallest shift of all. This indicates that all mutants have different and probably less tight conformation than that of the WT (as discussed above). Furthermore, it suggests that the inability to bind additional $\mathrm{Zn}^{2+}$ ions in the periphery of each monomer by H264 might lead to less stable closed conformation which results in smaller shift. Interestingly, D249H shows bigger shift than that of the WT, suggesting that although the 249 residues do not chelate the $\mathrm{Zn}^{2+}$ ions in the D249E/N mutants and $\mathrm{Cu}^{2+}$ in the WT Cu${ }^{2+}$-bound structure, the additional histidine residue in $\mathrm{D} 249 \mathrm{H}$ can help in the chelation of the $\mathrm{Zn}^{2+}$ to form a tighter conformation. This is supported by the fact that histidine residue tends to bind $\mathrm{Zn}^{2+}$ more than asparagine and glutamate ${ }^{4}$. In contrary, it might be that the bulky histidines lead to a different orientation of the monomers due to steric interference, and the bigger shift relates with a different swiveling movement of the monomers. All other mutants show smaller blue shift compared to the WT, implying the importance of every residue for proper metal binding.

MamM CTD mutant binding to $\mathrm{Ni}^{2+}$. For this study we solved the crystal structures of MamM CTD E289H and $\mathrm{D} 249 \mathrm{~N}$ with $\mathrm{Ni}^{2+}$, while we have previously solved the structure of the $\mathrm{Ni}^{2+}$-bound WT CTD ${ }^{16}$. All the $\mathrm{Ni}^{2+}$-bound structures exhibit the same conformation as the WT apo, with the $\mathrm{Ni}^{2+}$ ions bound only by each monomer's H264 residue and water molecules (Fig. 6). Previous PELDOR, Trp-fluorescence and ITC results of WT with $\mathrm{Ni}^{2+}$ suggested that $\mathrm{Ni}^{2+}$ binding is similar to that of $\mathrm{Zn}^{2+}$, where one ion is bound in the central site and two additional ions are bound to the peripheral sites, leading to a tight, rigid conformation as compared to the apo protein ${ }^{16}$. Since the $\mathrm{Ni}^{2+}$-bound WT CTD crystal structure did not show the conformational changes expected from the studies in solution, we cannot conclude from the mutants' crystal structures whether the mutations impact $\mathrm{Ni}^{2+}$-binding-dependent conformational changes. Contrary to the crystal structures, the Trpfluorescence quenching patterns of the different mutant proteins with $\mathrm{Ni}^{2+}$ (Fig. 2D, lower panel) show that each protein construct exhibits different binding abilities in solution. For example, the D249N, D249A-H264A and D249A-H285A show almost no quenching with the addition of $\mathrm{Ni}^{2+}$, suggesting that the D249 residue is important for $\mathrm{Ni}^{2+}$ binding near the W247 residues. D249H and D249H-H285D both show more quenching than that of the WT, and D249E shows less quenching, overall suggesting that it is not necessarily the aspartate in this location but that also histidine at the 249 position can bind $\mathrm{Ni}^{2+}$ in the central site. This is in agreement with the marked tendency of $\mathrm{Ni}^{2+}$ binding to histidine residues in crystal structures ${ }^{4}$. The E289H and E289HH264E both show much less quenching compared to the WT, while E289D shows similar pattern to that of the WT. These Trp-fluorescence results demonstrate that the negative charge at position 289 is important for proper chelation in a distant central site, which also gives an indication of allostery between both sites as shown previously for $\mathrm{Zn}^{2+}$ binding ${ }^{22}$. H264E mutant, which was shown by the $\mathrm{Zn}^{2+}, \mathrm{Cd}^{2+}$ and $\mathrm{Cu}^{2+} \mathrm{Trp}$-fluorescence results to influence the binding and the correct conformational changes, shows the same pattern as the WT protein for $\mathrm{Ni}^{2+}$. This was a surprising result because the $\mathrm{H} 264$ residue is the only $\mathrm{Ni}^{2+}$-chelating residue in all structures, thereby strengthening evidence which points to this residue as an important factor for DDMC attraction. When considering all these results and the similarity between $\mathrm{Zn}^{2+}$ and $\mathrm{Ni}^{2+}$ binding in the WT CTD, we suggest that 


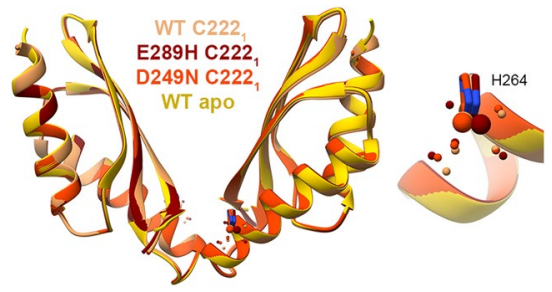

Figure 6. Crystal structures of different MamM CTD constructs bound to $\mathrm{Ni}^{2+}:$ MamM CTD WT with $\mathrm{Ni}^{2+}$ (SG C222 ${ }_{1}$, sandy brown, pdb code: $6 \mathrm{GMV}^{16}$ ), MamM CTD D249N with $\mathrm{Ni}^{+}$(SG C222 ${ }_{1}$, orange red, pdb code: $6 \mathrm{H} 8 \mathrm{D})$ and MamM CTD E289H with $\mathrm{Ni}^{2+}$ (SG C222 ${ }_{1}$, dark red, pdb code: $6 \mathrm{H} 81$ ) overlaid onto apo MamM CTD WT structure (SG C2221, gold, pdb code: $3 \mathrm{~W}^{2} \mathrm{X}^{14}$ ). All structures show the same conformation as the apo MamM CTD and bind $\mathrm{Ni}^{2+}$ by $\mathrm{H} 264$ and water molecules (right: enlarged binding site).

for $\mathrm{Ni}^{2+}$ the $\mathrm{H} 264$ position is not crucial for facilitation of the $\mathrm{Ni}^{2+}$ chelation in the central site-which would lead to the signal quenching-but only for the proper binding in the peripheral sites and the related conformational changes.

Unbound MamM CTD mutants and the special case of $\mathrm{Mn}^{2+}$. We solved the unbound structures of some MamM CTD mutants. All of them were crystallized in the presence of $\mathrm{Mn}^{2+}$; however, the $\mathrm{Mn}^{2+}$ could not be detected in their electron density map. MamM CTD H285D, D249H-H285D, H264E and H264E-E289H structures exhibit the same conformation as the WT apo form although they were co-crystallize with $\mathrm{Mn}^{2+}$ (Fig. 7A). However, E289D and D249N exhibit much tighter conformation compared to the apo form, which differs from both structures (Fig. 7B). As discussed above, one of the E289D dimers that could be built from the asymmetric unit monomers contained a weak manganese density, bound to $\mathrm{H} 264$ and water molecule; as the $\mathrm{Mn}^{2+}$ is bound solely at this position and could not be detected in the other dimer that adopts the same conformation, we assume that the $\mathrm{Mn}^{2+}$ is bound nonspecifically in this position and does not influence the conformational change. The reasons for the tighter conformation in both E289D and D249N apo forms were discussed above; both cases are great examples for how a single mutation can impact the dynamics of the apo forms, and potentially the regulation of the protein.

It was previously shown by Trp-fluorescence spectrometry, ITC and PELDOR that $\mathrm{Mn}^{2+}$ is the only DDMC out of six examined that MamM CTD WT protein could not bind ${ }^{16}$. We speculated that some mutants used in this study would show some binding abilities, as the binding site residues fit $\mathrm{Mn}^{2+}$ binding $\left(\mathrm{Mn}^{2+}\right.$ has high Asp, Glu and His binding propensities), $\mathrm{Mn}^{2+}$ is similar to the other DDMCs in its properties and many CDF proteins can transport $\mathrm{Mn}^{2+4}$. Surprisingly, we could not detect $\mathrm{Mn}^{2+}$ in any of the crystal structures crystallized in its presence (except E289D where the $\mathrm{Mn}^{2+}$ is bound nonspecifically), and moreover, all mutants showed no Trpfluorescence quenching (although paramagnetic) or blue shift in the presence of $\mathrm{Mn}^{2+}$ (Fig. 2C). Generally, the affinity of the first-row DDMC to any given substrate is following the Irving-Williams series that ranks the relative stability of first-row DDMCs' complexes: $\mathrm{Mn}^{2+}<\mathrm{Fe}^{2+}<\mathrm{Co}^{2+}<\mathrm{Ni}^{2+}<\mathrm{Cu}^{2+}>\mathrm{Zn}^{2+}$, meaning that $\mathrm{Cu}^{2+}$ and $\mathrm{Zn}^{2+}$ form more stable complexes with varied ligands, compared to $\mathrm{Mn}^{+}$and $\mathrm{Fe}^{2+}$ (this is usually correlated inversely with the cellular concentrations of the DDMCs in cells, in order to prevent the strong binding ions replacing the weaker binding ones in proteins) ${ }^{2,24}$, which can explain the lack of $\mathrm{Mn}^{2+}$ binding even in mutant forms. However, $\mathrm{Mn}^{2+}$ and $\mathrm{Fe}^{2+}$ are similar in their chemical properties including their size, coordination geometry and chelating residues type. Yet, $\mathrm{Mn}^{2+}$ is effectively discriminated by an unknown mechanism compared to iron which is the native metal that can bind and transported by $\mathrm{MamM}^{16}$. Interestingly, it was shown that uncultivated magnetotactic bacteria which are exposed to high concentrations of manganese, can incorporate it into the iron-based magnetic particles ${ }^{25}$. However, although $\mathrm{Mn}^{2+}$ has great affinity to magnetite when it abiotically synthesized, it is 100 times less concentrated in magnetotactic bacterial magnetite particles, indicating it is being discriminated in the process of transport into the magnetosome ${ }^{17}$. We previously suggested that to avoid overflow of manganese in the magnetosomes, as $\mathrm{Mn}^{2+}$ is more soluble in the bacteria cytosol (iron is mostly bound and stored to avoid the Fenton reaction ${ }^{26}$ ), and to avoid a sequential incorporation of it into magnetite particles, MTB developed a sophisticated mechanism for selectivity against manganese by the CTD. Here we further speculate that the $\mathrm{Mn}^{2+}$ selectivity is not resulted from direct interactions with the metal-binding residues, as we can see that mutations could not affect this discrimination. It is most likely that it involves residues in the second and third sphere around the metal which influence the general electrostatic and binding affinities in a way we cannot easily detect. Yet, we do not exclude the chance that other binding-site mutants would change the observed selectivity.

\section{Conclusion}

The DDMC binding site composition in MamM CTD impacts its ability to bind different DDMCs as well as impacting the bound-state conformation. The repertoire of bound conformations observed by the crystal structures is narrow, with all structures exhibiting the same or tighter conformation than the WT CTD. Furthermore, there are only a few specific residues that can participate in metal chelation by all mutant forms (as the DDMCs were not necessarily chelated by the binding site residues but also by other residues; Fig. 8). These findings indicate that although we can alter the DDMC binding properties by mutating the binding site residues, in terms of conformation, identity of the binding sites and the number of metal bound to the CTD, the CTD conformational 


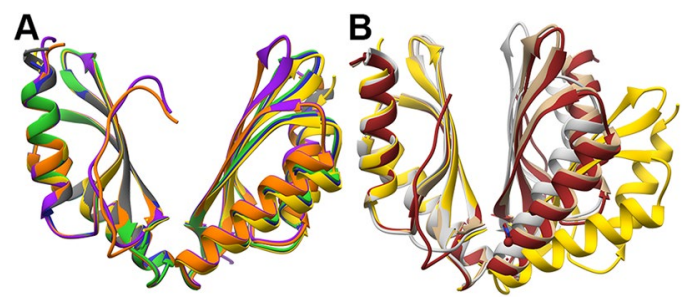

Figure 7. Crystal structures of different MamM CTD constructs that were crystallized in the presence of $\mathrm{Mn}^{2+}$. (A) Crystal structures of MamM CTD H285D (SG C222 ${ }_{1}$, medium blue, pdb code: 6H8I), MamM CTD D249H-H285D (SG C222 ${ }_{1}$, lime green, pdb code: 6HA2), MamM CTD H264E (SG C222 1 , dim gray, pdb code: $6 \mathrm{H} 5 \mathrm{~V}$ ) and MamM CTD H264E-E289H (SG P2 $2_{1} 2_{1}$, chains A + D in orange, chains B + C in purple, pdb code: $6 \mathrm{HAN}$ ) overlaid onto apo MamM CTD WT structure (SG C2221, gold, pdb code: $3 \mathrm{~W} 5 \mathrm{X}^{14}$ ). All crystals were formed in the presence of $\mathrm{Mn}^{2+}$, however it could not be detected in the electron density map. Structures of all mutants have similar conformation to that of the WT apo. (B) Crystal structures of MamM CTD D249N (SG C222 ${ }_{1}$, dim gray, pdb code: $6 \mathrm{H} 88$ ), and MamM CTD E289D (SG P6 22 , chains A + A' in brown, chains B + C in tan, pdb code: $6 \mathrm{H} 9 \mathrm{P}$ ) overlaid onto apo MamM CTD WT structure (SG C2221, gold, pdb code: 3 W5 $\mathrm{X}^{14}$ ). Both crystals were formed in the presence of $\mathrm{Mn}^{2+}$, however it could be detected in the electron density map of only one of the MamM CTD E289D dimers, chelated by only one residue, H264, and one water molecule. Both mutant structures exhibit tighter dimerization compared to the apo MamM CTD WT, yet the conformation is different between them.

space and the possible binding sites are limited for only specific stable combinations. The CTD can control the metal selectivity of the overall protein by reacting distinctively, in terms of structural changes, to the different DDMCs. However, discrimination against $\mathrm{Mn}^{2+}$ was not changed although binding residue were mutated. This may indicate that for many metal binding proteins, the metal selectivity can be determined by other means such as recognition with metal chelator or metal metallochaperones that bring the metal to the binding site, or by larger residue set which may include a second or third residue sphere around the binding site. Although the CTD can bind various metals, only in response to some DDMCs can it adopt the exact tight conformation that will facilitate the sequential conformational change of the TMD that results in metal transport. The mutations can alter the conformational changes so that different monomer orientation or degree-of-closure of the CTD dimer are achieved in the presence of specific DDMCs (if at all) so as to preserve, promote or block the actual transport. As these alterations putatively regulate the overall protein function, future studies will be able to use our results to examine how these mutations impact the whole protein selectivity, as it relates to the composition of the transport site in the transmembrane domain as well ${ }^{4,10,27}$, and to design specific mutations in MamM CTD in vivo to facilitate a controlled and specific DDMC binding to the CTD and, in combination with mutation in the transmembrane transport site, to insert DDMCs selectively into the magnetosome to form magnetic particles with varied magnetic properties.

\section{Methods}

Site-directed mutagenesis and protein expression. mamM CTD gene from Magnetospirillum gryphiswaldense MSR-1 (UniProt Q6NE57 residues 215-318) was previously cloned into pET28a(+) vector (Novagen, Merck Biosciences, Germany $)^{28}$; in this construct, pET28a-MamM-CTD-MSR1, the mamM gene was fused in-frame to express a six-His tag at the N-terminus of the protein followed by a thrombin proteolysis site. All MamM CTD mutations were applied to the pET28a-MamM-CTD-MSR1 vector using the QuickChange sitedirected mutagenesis method (Stratagene, La Jolla, CA, USA). Primers containing single mutation sites (Hylabs, Rehovot, Israel) were designed and used for PCR amplifications. All MamM CTD forms were expressed similarly to MamM CTD WT, as previously described ${ }^{28}$.

Protein purification. All MamM CTD constructs were purified as previously described for MamM CTD M250L and other mutants ${ }^{21,22,28}$. For all experiments, protein concentration was determined by measuring protein absorption at $280 \mathrm{~nm}$.

Crystallization and structure determination. Purified MamM CTD constructs at $20 \mathrm{mg} \mathrm{mL}^{-1}$ concentration in buffer containing $10 \mathrm{mM}$ Tris $\mathrm{pH}=8.0,150 \mathrm{mM} \mathrm{NaCl}, 5 \mathrm{mM} \beta$-mercaptoethanol and $3.375 \mathrm{mM}$ metal solution $\left(\mathrm{ZnCl}_{2}, \mathrm{CdCl}_{2}, \mathrm{MnCl}_{2}, \mathrm{NiCl}_{2}, \mathrm{FeCl}_{2}\right.$ or $\left.\mathrm{CuSO}_{4}\right)$, were subjected to crystallization trials using the vapor diffusion method at $293 \mathrm{~K}(0.3 \mu \mathrm{L}$ protein with $0.3 \mu \mathrm{L}$ reservoir solution for all protein-metal pairs). Crystals were harvested with or without treatment of cryo agent and flash-frozen in liquid nitrogen. Data collection was performed on a single-crystal at a temperature of $100 \mathrm{~K}$. For all structures, data reduction was performed with $\mathrm{XDS}^{29}$ and data scaling with Aimless ${ }^{30}$ (after Aimless scaling, data of H264E Cd ${ }^{2+}$ form2, H264E-E289H, E289D $\mathrm{Mn}^{2+}$ form and E289D Cd ${ }^{2+}$ form were further processed with the STARANISO server (Global Phasing Ltd., Cambridge, UK)). Phases were obtained by the molecular replacement method with the MamM CTD WT structure (PDB code: $3 \mathrm{~W} 5 \mathrm{X}^{14}$ ) as a template, using Phaser $\mathrm{MR}^{31}$. All structures were refined by Refmac $5^{32}$, Phenix ${ }^{33}$ and/or PDB_REDO server ${ }^{34}$, while manual refinement was conducted using Coot version 0.8.9 ${ }^{35}$. Aimless, Phaser MR and Refmac5 were used through the CCP4i package ${ }^{36}$. Rfree calculation used $5 \%$ of the data. All 


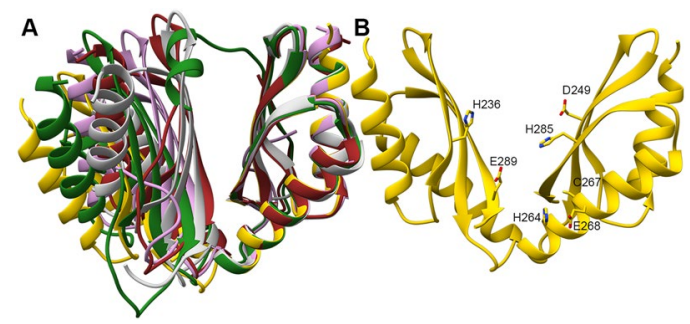

Figure 8. All detected MamM CTD conformations and metal-binding residues. (A) Crystal structures of MamM CTD WT with $\mathrm{Cu}^{2+}$ (SG P22 $2_{1}$, forest green, pdb code: 6GP6 ${ }^{16}$ ), MamM CTD D249N with no metal bound $\left(\mathrm{Mn}^{2+}\right.$ in crystallization condition, light gray, pdb code: $\left.6 \mathrm{H} 88\right)$ and with $\mathrm{Zn}^{2+}$ (SG P3 121 , oink, pdb code: 6H87), and MamM CTD E289D with $\mathrm{Mn}^{+}$(SG P6 22 , chains A + A', brown, pdb code: 6H9P) overlaid onto apo MamM CTD WT structure (SG C222, gold, pdb code: $3 \mathrm{~W} 5 \mathrm{X}^{14}$ ). These structures represent all the detected conformation of MamM CTD WT and mutants in the presence and absence of different metals. (B) Apo MamM CTD WT structure (pdb code: $3 W_{5} \mathrm{X}^{14}$ ). All metal-binding residues that were detected in all MamM CTD crystal structures are indicated (D245 and E282 that bind $\mathrm{Cd}^{2+}$ in the MamM CTD E289D-Cd ${ }^{2+}$ structure are not shown as they are biologically irrelevant; for clarity, each residue is shown only in one monomer).

crystallization and cryo conditions, data collection details, data collection and refinement statistics and the used refinement software are given in Tables S1-S3.

Least-squares overlays. All MamM CTD construct structures were overlaid and all figures were prepared using UCSF Chimera package, version $1.12^{37}$.

Fluorescence spectrometry. Changes in tryptophan intrinsic fluorescence were recorded using Fluorolog-3 (Horiba Scientific, Edison, NJ, USA) equipped with quartz cell with $1 \mathrm{~cm}$ optical path length at ambient temperature. Samples of $1 \mathrm{~mL}$ MamM CTD proteins at $5 \mu \mathrm{M}$ concentration in buffer containing $10 \mathrm{mM}$ Tris $\mathrm{pH}=8.0$ and $150 \mathrm{mM} \mathrm{NaCl}$, were titrated using $2.5 \mathrm{mM}$ metal solution in the same buffer to reach different concentrations $\left(\mathrm{ZnCl}_{2}, \mathrm{CdCl}_{2}, \mathrm{NiCl}_{2}, \mathrm{MnCl}_{2}, \mathrm{CuSO}_{4}\right)$. Samples were measured at $\lambda \mathrm{ex} 297 \mathrm{~nm}$, and the emission spectrum for each metal concentration was recorded at 310-450 nm. For each metal, the titration was replicated three times, and each spectrum was fitted to Extreme function by OriginPro (R-Square (COD) $>0.98$ ) (OriginLab Corporation, Northampton, MA, USA). The maximum wavelength (wavelength at maximum intensity) and the intensity at that wavelength (aka maximum intensity) were averages for each metal concentration. Error is reported as the standard deviation. WT data is adapted from Barber-Zucker et al. $2020^{16}$, where detailed analysis of the fluorescence data curves for each metal is given. As $\mathrm{Cd}^{2+}$ and $\mathrm{Zn}^{2+}$ mainly cause concentration-dependent clear spectral shift and $\mathrm{Ni}^{2+}$ and $\mathrm{Cu}^{2+}$ concentration-dependent signal quenching, to simplify the analysis we discuss $\mathrm{Cd}^{2+}$ and $\mathrm{Zn}^{2+}$ impact on the spectral shift (hence their impact on the conformational change) and $\mathrm{Ni}^{2+}$ and $\mathrm{Cu}^{2+}$ impact on the signal quenching (hence their ability to be bound closely to the W247 residue rather than their direct impact on the conformational change). The addition of $\mathrm{Mn}^{2+}$ did not result in signal quenching or spectral shift in any of the MamM CTD mutant constructs; hence, the Trp-fluorescence results with $\mathrm{Mn}^{2+}$ are not discussed in the Results and Discussion section.

\section{Data availability}

MamM CTD structures have been deposited in the Protein Data Bank under the following accession codes: 6H5V, 6H5M, 6H5U, 6H8G, 6HAO, 6H88, 6H87, 6H8A, 6H89, 6H8D, 6H5K, 6H9Q, 6H84, 6H83, 6HA2, 6H8I, $6 \mathrm{H} 9 \mathrm{~T}, 6 \mathrm{H} 81,6 \mathrm{HAN}, 6 \mathrm{H} 85$, 6H9P, 6HHS. All other dataset generated during the current study are available from the corresponding author on reasonable request.

Received: 4 May 2020; Accepted: 29 July 2020

Published online: 20 August 2020

\section{References}

1. Lyons, T. J., Eide, D. J. \& Introduction, V. Transport and storage of metal ions in biology. In Biologocal Inorganic Chemistry: Structure and Reactivity (eds Bertini, I. et al.) 57-78 (University Science Books, Mill Valley, 2006).

2. Dudev, T. \& Lim, C. Competition among metal ions for protein binding sites: Determinants of metal ion selectivity in proteins. Chem. Rev. 114, 538-556 (2014).

3. Haney, C. J., Grass, G., Franke, S. \& Rensing, C. New developments in the understanding of the cation diffusion facilitator family. J. Ind. Microbiol. Biotechnol. 32, 215-226 (2005).

4. Barber-Zucker, S., Shaanan, B. \& Zarivach, R. Transition metal binding selectivity in proteins and its correlation with the phylogenomic classification of the cation diffusion facilitator protein family. Sci. Rep. 7, 16381 (2017).

5. Kolaj-Robin, O., Russell, D., Hayes, K. A., Pembroke, J. T. \& Soulimane, T. Cation diffusion facilitator family: Structure and function. FEBS Lett. 589, 1283-1295 (2015).

6. Cubillas, C., Vinuesa, P., Tabche, M. L. \& García-de los Santos, A. Phylogenomic analysis of cation diffusion facilitator proteins uncovers $\mathrm{Ni}^{2+} / \mathrm{Co}^{2+}$ transporters. Metallomics 5, 1634-1643 (2013).

7. Montanini, B., Blaudez, D., Jeandroz, S., Sanders, D. \& Chalot, M. Phylogenetic and functional analysis of the cation diffusion facilitator (CDF) family: Improved signature and prediction of substrate specificity. BMC Genomics 8, 107 (2007). 
8. Lu, M. \& Fu, D. Structure of the zinc transporter YiiP. Science 317, 1746-1748 (2007).

9. Lasry, I. et al. In situ dimerization of multiple wild type and mutant zinc transporters in live cells using bimolecular fluorescence complementation. J. Biol. Chem. 289, 7275-7292 (2014).

10. Uebe, R. et al. The cation diffusion facilitator proteins MamB and MamM of Magnetospirillum gryphiswaldense have distinct and complex functions, and are involved in magnetite biomineralization and magnetosome membrane assembly. Mol. Microbiol. 82, 818-835 (2011).

11. Lu, M., Chai, J. \& Fu, D. Structural basis for autoregulation of the zinc transporter YiiP. Nat. Struct. Mol. Biol. 16, 1063-1067 (2009).

12. Lopez-Redondo, M. L., Coudray, N., Zhang, Z., Alexopoulos, J. \& Stokes, D. L. Structural basis for the alternating access mechanism of the cation diffusion facilitator YiiP. Proc. Natl. Acad. Sci. USA 115, 3042-3047 (2018).

13. Cherezov, V. et al. Insights into the mode of action of a putative zinc transporter CzrB in Thermus thermophilus. Structure 16, 1378-1388 (2008).

14. Zeytuni, N. et al. Cation diffusion facilitators transport initiation and regulation is mediated by cation induced conformational changes of the cytoplasmic domain. PLoS ONE 9, e92141 (2014).

15. Cotrim, C. A., Jarrott, R. J., Martin, J. L. \& Drew, D. A structural overview of the zinc transporters in the cation diffusion facilitator family. Acta Crystallogr. Sect. D Struct. Biol. 75, 357-367 (2019).

16. Barber-Zucker, S. et al. The cation diffusion facilitator protein MamM's cytoplasmic domain exhibits metal-type dependent binding modes and discriminates against $\mathrm{Mn}^{2+}$. bioRxiv https://doi.org/10.1101/2020.02.02.930644 (2020).

17. Amor, M. et al. Chemical signature of magnetotactic bacteria. Proc. Natl. Acad. Sci. USA 112, 1699-1703 (2015).

18. Barber-Zucker, S. \& Zarivach, R. A look into the biochemistry of magnetosome biosynthesis in magnetotactic bacteria. ACS Chem. Biol. 12, 13-22 (2017).

19. Uebe, R. \& Schüler, D. Magnetosome biogenesis in magnetotactic bacteria. Nat. Rev. Microbiol. 14, 621-637 (2016).

20. Faivre, D. \& Schüler, D. Magnetotactic bacteria and magnetosomes. Chem. Rev. 108, 4875-4898 (2008).

21. Barber-Zucker, S. et al. Disease-homologous mutation in the cation diffusion facilitator protein MamM causes single-domain structural loss and signifies its importance. Sci. Rep. 6, 31933 (2016).

22. Barber-Zucker, S. et al. Metal binding to the dynamic cytoplasmic domain of the cation diffusion facilitator (CDF) protein MamM induces a 'locked-in' configuration. FEBS J. 286, 2193-2215 (2019).

23. Lang, C. \& Schüler, D. Biogenic nanoparticles: Production, characterization, and application of bacterial magnetosomes. J. Phys. Condens. Matter 18, S2815-S2828 (2006).

24. Irving, H. \& Williams, R. J. P. Order of stability of metal complexes. Nature 162, 746-747 (1948).

25. Keim, C. N., Lins, U. \& Farina, M. Manganese in biogenic magnetite crystals from magnetotactic bacteria. FEMS Microbiol. Lett. 292, 250-253 (2009).

26. Chandrangsu, P., Rensing, C. \& Helmann, J. D. Metal homeostasis and resistance in bacteria. Nat. Rev. Microbiol. 15, 338-350 (2017).

27. Hoch, E. et al. Histidine pairing at the metal transport site of mammalian $\mathrm{ZnT}$ transporters controls $\mathrm{Zn}^{2+}$ over Cd$^{2+}$ selectivity. Proc. Natl. Acad. Sci. USA 109, $7202-7207$ (2012).

28. Zeytuni, N., Offer, T., Davidov, G. \& Zarivach, R. Crystallization and preliminary crystallographic analysis of the C-terminal domain of MamM, a magnetosome-associated protein from Magnetospirillum gryphiswaldense MSR-1. Acta Crystallogr. Sect. F Struct. Biol. Cryst. Commun. 68, 927-930 (2012).

29. Kabsch, W. XDS. Acta Crystallogr. D Biol. Crystallogr. 66, 125-132 (2010).

30. Evans, P. R. \& Murshudov, G. N. How good are my data and what is the resolution?. Acta Crystallogr. D Biol. Crystallogr. 69, 1204-1214 (2013).

31. McCoy, A. J. et al. Phaser crystallographic software. J. Appl. Crystallogr. 40, 658-674 (2007).

32. Murshudov, G. N. et al. REFMAC5 for the refinement of macromolecular crystal structures. Acta Crystallogr. D Biol. Crystallogr. 67, 355-367 (2011).

33. Adams, P. D. et al. PHENIX: A comprehensive Python-based system for macromolecular structure solution. Acta Crystallogr. D Biol. Crystallogr. 66, 213-221 (2010).

34. Joosten, R. P., Long, F., Murshudov, G. N. \& Perrakis, A. The PDB_REDO server for macromolecular structure model optimization. IUCrJ 1, 213-220 (2014).

35. Emsley, P., Lohkamp, B., Scott, W. G. \& Cowtan, K. Features and development of Coot. Acta Crystallogr. D Biol. Crystallogr. 66, 486-501 (2010).

36. Winn, M. D. et al. Overview of the CCP4 suite and current developments. Acta Crystallogr. D Biol. Crystallogr. 67, 235-242 (2011).

37. Pettersen, E. F. et al. UCSF Chimera-A visualization system for exploratory research and analysis. J. Comput. Chem. 25, 1605-1612 (2004).

\section{Acknowledgments}

SBZ and RZ are supported by the Israel Ministry of Science, Technology and Space, the Israel Science Foundation (Grant No. 167/16), the European Molecular Biology Organization and CMST COST Action CM1306.

\section{Author contributions}

S.B.Z. and R.Z. designed the study and wrote the paper. S.B.Z. and A.S. performed the experiments. S.B.Z., A.S., S.K. and R.Z. designed the experiments, analyzed the results and approved the final version of the manuscript.

\section{Competing interests}

The authors declare no competing interests.

\section{Additional information}

Supplementary information is available for this paper at https://doi.org/10.1038/s41598-020-71036-4.

Correspondence and requests for materials should be addressed to R.Z.

Reprints and permissions information is available at www.nature.com/reprints.

Publisher's note Springer Nature remains neutral with regard to jurisdictional claims in published maps and institutional affiliations. 
(c) (i) Open Access This article is licensed under a Creative Commons Attribution 4.0 International cc) License, which permits use, sharing, adaptation, distribution and reproduction in any medium or format, as long as you give appropriate credit to the original author(s) and the source, provide a link to the Creative Commons license, and indicate if changes were made. The images or other third party material in this article are included in the article's Creative Commons license, unless indicated otherwise in a credit line to the material. If material is not included in the article's Creative Commons license and your intended use is not permitted by statutory regulation or exceeds the permitted use, you will need to obtain permission directly from the copyright holder. To view a copy of this license, visit http://creativecommons.org/licenses/by/4.0/.

(C) The Author(s) 2020 